\title{
Variations in the posterior division branches of the mandibular nerve in human cadavers
}

\author{
Balaji Thotakura ${ }^{1}$, MSc, PhD, Sharmila Saran $\underline{\text { Rajendran }}^{1}$, MSc, Vaithianathan $\underline{G n a n a s u n d a r a m}^{1}$, MSc,
} Aruna Subramaniam ${ }^{1}$, MBBS, MS

\begin{abstract}
INTRODUCTION The lingual, inferior alveolar and auriculotemporal nerves, being branches of the posterior division of the mandibular nerve, mainly innervate the mandibular teeth and all the major salivary glands. Anomalous communications among these branches are widely reported due to their significance to various treatment procedures undertaken in the region. This study was performed as detailed exploration of the functional perspectives of such communicating branches would further enhance the scope of these procedures.

METHODS A total of 36 specimens were dissected to examine the infratemporal region. The branches from the posterior division of the mandibular nerve - namely the lingual, inferior alveolar and auriculotemporal nerves - were carefully dissected, and their branches were studied and analysed for abnormal course.

RESULTS Communication between branches of the posterior division of the mandibular nerve was observed in four specimens. In two of the four specimens, communication between the mylohyoid and lingual nerves was observed. A rare and seldom reported type of communication between the auriculotemporal and inferior alveolar nerves is described in this study. This communicating nerve split into two to form a buttonhole for the passage of the mylohyoid nerve.

CONCLUSION Such communicating branches between nerves found in this study are developmental in origin and thought to maintain functional integrity through an alternative route.
\end{abstract}

Keywords: auriculotemporal, inferior alveolar, lingual, mandibular, mylohyoid

\section{INTRODUCTION}

The mandibular division of the trigeminal nerve contains both sensory and motor fibres, while its maxillary and ophthalmic divisions constitute predominantly sensory fibres. The mandibular nerve, immediately after its exit from the skull through the foramen ovale, divides into the anterior and posterior branches. Branches of the posterior division of the mandibular nerve namely the lingual nerve, inferior alveolar nerve (IAN) and auriculotemporal nerve (ATN) - are important, as they innervate the structures involved in mastication, salivation, speech and taste sensations. ${ }^{(1)}$ In contrast, the branches of the anterior division of the mandibular nerve are predominantly muscular.

Variations in the branching pattern and abnormal communication among the lingual nerve, ATN and IAN are extensively documented. ${ }^{(2,3)}$ The existence of communication between the lingual nerve and IAN, which is a frequently reported variation in the literature, ${ }^{(3)}$ is thought to be responsible for inadequate mandibular anaesthesia. ${ }^{(4)}$ The origin of the ATN by more than two roots and its relation to the maxillary artery, ${ }^{(5)}$ as well as communicating nerve twig between the ATN and IAN and its incidence, are also widely documented. ${ }^{(3)}$ However, communication between the mylohyoid nerve $(\mathrm{MHN})$ and lingual nerve is scarcely reported. ${ }^{(6)}$

Abnormal communications most commonly encountered among the posterior division branches of the mandibular nerve are from either studies on one particular branch or individual case reports. ${ }^{(7,8)}$ The present study focused on the communication among the posterior division branches of the mandibular nerve as a whole, as well as the significance and clinical implications of such abnormal communications. The authors also aimed to describe the purpose of such communication and its implications for various surgical procedures undertaken in the region.

\section{METHODS}

A total of 36 infratemporal regions - inclusive of both sides and irrespective of age and gender - were dissected for the present study. The lingual nerve, IAN and ATN arising from the posterior division of the mandibular nerve were carefully exposed. The course of the ATN was traced as far behind the neck of the mandible as possible. The IAN was followed up to the mandibular foramen, and further dissection was carried out by removing part of the mandible. The MHN branch of the IAN was traced up to the mylohyoid muscle. The lingual nerve was dissected until its entrance into the submandibular region.

\section{RESULTS}

Among the 36 specimens dissected, 4 (11.1\%) were observed to have variations in the posterior division branches of the mandibular nerve. Communication was observed between the MHN and lingual nerve in two specimens. In one of these two specimens, the communicating branch was found to be bilateral (Fig. 1). This branch was thicker than the MHN and found to arise from the MHN at the lower border of the lateral pterygoid muscle (Fig. 1). The branch coursed deep to the IAN to

${ }^{1}$ Department of Anatomy, Chettinad Hospital and Research Institute, Kanchipuram, India

Correspondence: Dr Thotakura Balaji, Professor, Department of Anatomy, Chettinad Hospital and Research Institute, Kanchipuram Dt 603103, India. balajitk@yahoo.com 

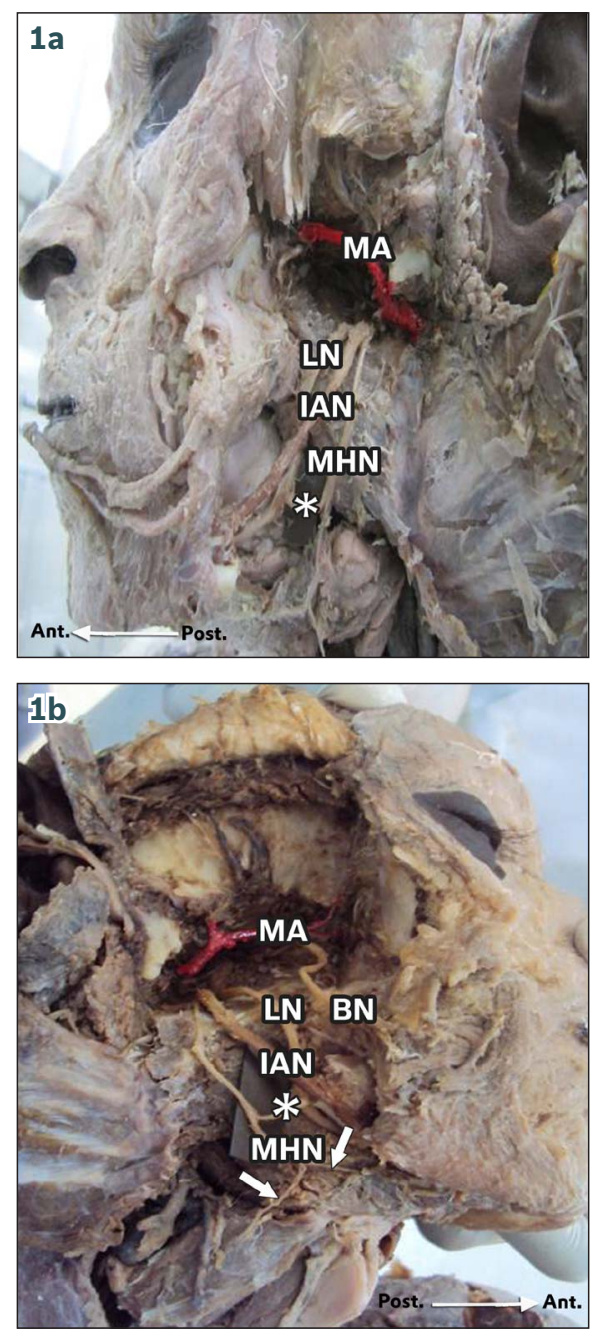

Fig. 1 Lateral views of the (a) left and (b) right infratemporal regions show a communicating branch (marked *) between the mylohoid and lingual nerves on both sides. The inferior alveolar nerve follows a normal course. An unusually thick branch of the buccal nerve, as well as branches to the mylohyoid and digastric muscles (arrows), is also seen on the right side. BN: buccal nerve; IAN: inferior alveolar nerve; LN: lingual nerve; MA: maxillary artery; MHN: mylohoid nerve

join the lingual nerve prior to its entrance into the submandibular region. Beyond the level of this communication, the course of the MHN was normal and supplied branches to the mylohyoid muscle and anterior belly of the digastric muscle (Fig. 1a). In the second specimen, a thin branch was found to connect the MHN and lingual nerve in the right infratemporal region.

Communication between the ATN and IAN was observed in the left infratemporal region of a third specimen. A branch from the trunk of the ATN was found to have descended anteriorly to join the posterior border of the IAN at an acute angle in this specimen (Fig. 2). Interestingly, this communicating branch, prior to its termination into the IAN, split into two to form a buttonhole communicating nerve loop for the passage of the MHN soon after the latter's origination from the IAN. The MHN passed though this communicating nerve loop (Fig. 2). The further courses of the IAN, ATN and MHN were found to be normal.

In the right infratemporal fossa of a fourth specimen, two communicating branches from the lower root of the ATN descended to join the IAN (Fig. 3). Even though the ATN originated

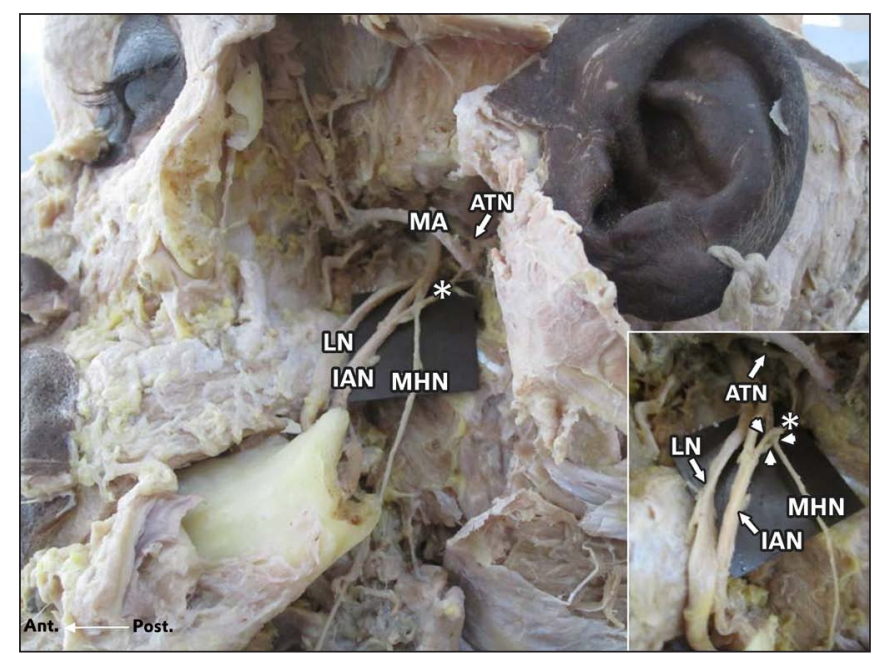

Fig. 2 Lateral view of the left infratemporal region shows a communicating branch (marked *) extending between the auriculotemporal nerve and inferior alveolar nerve. The mylohyoid nerve is posterior to the inferior alveolar nerve, while the lingual nerve is anterior to it. Inset shows a closer view of the mylohyoid nerve passing through the communicating loop (arrowheads) formed by the communicating branch (marked*) between the auriculotemporal and inferior alveolar nerves. ATN: auriculotemporal nerve; IAN: inferior alveolar nerve; LN: lingual nerve; MA: maxillary artery; MHN: mylohoid nerve

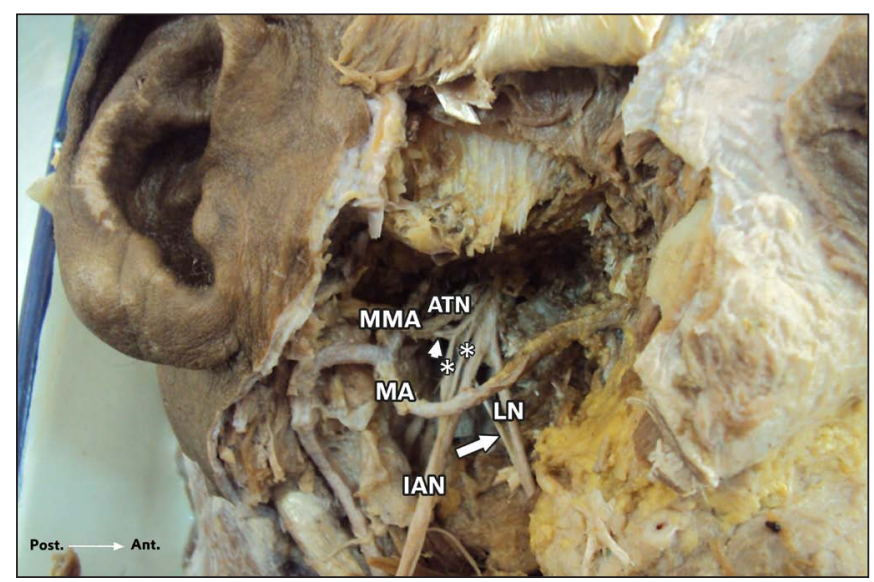

Fig. 3 Lateral view of the right infratemporal region shows two communicating branches (marked ${ }^{*}$ ) connecting the inferior root of the auriculotemporal nerve (arrowhead) with the inferior alveolar nerve. The middle meningeal artery from the maxillary artery lies lateral to the auriculotemporal nerve. The lingual nerve is anterior to the inferior alveolar nerve, and the chorda tympani nerve (arrow) joins the lingual nerve at an acute angle. ATN: auriculotemporal nerve; IAN: inferior alveolar nerve; LN: lingual nerve; MA: maxillary artery; MMA: middle meningeal artery

from two roots, it failed to form a buttonhole for the passage of the middle meningeal artery in this specimen (Fig. 3). Instead, the middle meningeal artery was found to be present lateral to the ATN. No other abnormalities were observed in the course of the lingual nerve, IAN and ATN.

\section{DISCUSSION}

Being the sole motor branch of the posterior division of the mandibular nerve, the MHN supplies the mylohyoid muscle and the anterior belly of the digastric muscle. The amount of literature on communication between the MHN and lingual nerve is minimal. ${ }^{(6)}$ The proximity of the lingual nerve to the third molar 
tooth makes it more susceptible to injury during oral and dental surgeries, ${ }^{(9)}$ although complete recovery from such an injury is common. ${ }^{(6)}$ The existence of communication between the MHN and lingual nerve might help in the recovery of lingual nerve injury by contributing to additional sensory innervations of the tongue. ${ }^{(6)}$ The existing literature mainly focuses on the clinical implications of such communicating branches, but fails to give a convincing explanation of its functional perspective. ${ }^{(10)}$

In the present study, a thick branch from the MHN joining the lingual nerve was found bilaterally in one specimen. Such bilateral branching is seldom reported. The mylohyoid muscle plays a vital role in deglutition and mastication. Deglutition is a complex process involving the muscles of the tongue, palate and pharynx, as well as the suprahyoid muscles. ${ }^{(11)}$ A communicating branch from the MHN to the lingual nerve is developmental in origin and assumed to convey proprioceptive fibres from the mylohyoid muscle through the lingual nerve. ${ }^{(12)}$ This communicating branch, if present, might be involved in the coordination of tongue movements with suprahyoid muscles via proprioceptive impulses.

It is not uncommon to find that the ATN arises from more than one root and does not always form a buttonhole around the middle meningeal artery. ${ }^{(13)}$ In fact, the origin of the ATN by up to four roots has been reported. ${ }^{(14)}$ The connections of the ATN with the IAN and facial nerve are well documented, ${ }^{(1)}$ while communication between the ATN and IAN is the most commonly reported. ${ }^{(4)}$ While a majority of secretomotor fibres arising from the otic ganglion are found in the ATN, some fibres are detected in other branches of the mandibular nerve. ${ }^{(15)}$ Of the two specimens in our study with communication between the ATN and IAN, one exhibited an unusual pattern where a single communicating branch from the ATN to IAN split into two to form a buttonhole for the passage of the MHN. The possibility of MHN compression in such scenarios cannot be completely excluded. This type of nerve communication is seldom reported and such communicating branches are thought to convey postganglionic fibres from the otic ganglion to the IAN through the ATN. The most convincing explanation for such communication is likely the hitchhiking of fibres during the developmental process. ${ }^{(16)}$ Such fibres, passing via the IAN, are thought to innervate the lower labial glands. Studies on animal experiments have also proven that the ATN, MHN and mental nerve have the same central projections in the trigeminal nucleus. ${ }^{(17)}$
In conclusion, aberrant nerve communications are not uncommon, and knowledge regarding the functional perspectives of such communicating branches is absolutely necessary in order to effectively perform surgical procedures in any part of the human body. The presence of such communicating nerves among the posterior division branches of the mandibular nerve is thought to serve as an alternative route for maintaining the functional integrity of the structures innervated by it.

\section{REFERENCES}

1. Berkovitz BK. Infratemporal region and temporomandibular joint. In: Standring S, ed. Gray's Anatomy: The Anatomical Basis of Clinical Practice. 39th ed. Edinburgh: Elsevier Churchill Livingstone, 2005: 526-30.

2. Siéssere S, Hallak Regalo SC, Semprini M, et al. Anatomical variations of the mandibular nerve and its branches correlated to clinical situations. Minerva Stomatol 2009; 58:209-15.

3. Rácz L, Maros T. [The anatomic variants of the lingual nerve in human.] Anat Anz 1981; 149:64-71. German.

4. Anil A, Peker T, Turgut HB, Gülekon IN, Liman F. Variations in the anatomy of the inferior alveolar nerve. Br J Oral Maxillofac Surg 2003; 41:236-9.

5. Baumel JJ, Vanderheiden JP, McElenney JE. The auriculotemporal nerve of man. Am J of Anat 1971; 130:431-40.

6. Fazan VPS, Rodrigues Filho OA, Matamala F. Communication between the mylohyoid and lingual nerves: clinical implications. Int J Morphol 2007; 25:561-4.

7. Kocabiyik N, Varol A, Sencimen M, Ozan H. An unnamed branch of the lingual nerve: gingival branch. Br J Oral Maxillofac Surg 2009; 47:214-7.

8. Madeira MC, Percinoto C, das Graças M Silva M. Clinical significance of supplementary innervation of the lower incisor teeth: a dissection study of the mylohyoid nerve. Oral Surg Oral Med Oral Pathol 1978; 46:608-14.

9. Joshi A, Rood JP. External neurolysis of the lingual nerve. Int J Oral Maxillofac Surg 2002; 31:40-3.

10. Potu BK, Pulakunta T, Ray B, et al. Unusual communication between the lingual nerve and mylohyoid nerves in a South Indian male cadaver: its clinical significance. Rom J Morphol Embryol 2009; 50:145-6.

11. Mascaro MB, Picoli LC, Santos FM, et al. Anatomical variation of the anterior belly of the digastric muscle: case report and clinical implications. J Morphol Sci 2011; 28:72-5.

12. Furusawa K, Yamaoka M, Fujimoto K, Kumai T. Role of proprioceptors in the mylohyoid muscle. Brain Res Bull 1994; 35:233-6.

13. Roy TS, Sarkar AK, Panicker HK. Variation in the origin of the inferior alveolar nerve. Clin Anat 2002; 15:143-7.

14. Gülekon N, Anil A, Poyraz A et al. Variations in the anatomy of the auriculotemporal nerve. Clin Anat 2005; 18:15-22.

15. Pai MM, Swamy RS, Prabhu LV. A variation in the morphology of the inferior alveolar nerve with potential clinical significance. Biomed Int 2010; 1:93-5.

16. Segade LA, Suarez Quintanilla D, Suarez Nuñez JM. The postganglionic parasympathetic fibers originating in the otic ganglion are distributed in several branches of the trigeminal mandibular nerve: an HRP study in the guinea pig. Brain Res 1987; 411:386-90.

17. Takemura M, Sugimoto T, Sakai A. Topographic organization of central terminal region of different sensory branches of the rat mandibular nerve. Exp Neurol 1987; 96:540-57. 